\title{
A Synchronous Motor Imagery Based Neural Physiological Paradigm for Brain Computer Interface Speller
}

\author{
Lei Cao ${ }^{1,2 \dagger}$, Bin Xia ${ }^{1,2 * t}$, Oladazimi Maysam ${ }^{3}$, Jie $\mathrm{Li}^{4}$, Hong Xie ${ }^{1}$ and Niels Birbaumer ${ }^{2,5}$ \\ ${ }^{1}$ Department of Computer Science, College of Information Engineering, Shanghai Maritime University, Shanghai, China, \\ ${ }^{2}$ Institute of Medical Psychology and Behavioral Neurobiology, University of Tuebingen, Tuebingen, Germany, ${ }^{3}$ Werner \\ Reichardt, Center for Integrative Neuroscience (System Neurophysiology), University of Tuebingen, Tuebingen, Germany, \\ ${ }^{4}$ Department of Computer Science and Technology, Tongji University, Shanghai, China, ${ }^{5}$ IRCCS Fondazione Ospedale San \\ Camillo, Venezia, Italy
}

OPEN ACCESS

Edited by:

John H. Gruzelier,

Goldsmiths, University of London,

United Kingdom

Reviewed by:

Noman Naseer,

Air University, Pakistan

Hasan Ayaz,

Drexel University, United States

*Correspondence:

Bin Xia

xawen267@gmail.com

${ }^{\dagger}$ These authors have contributed equally to this work.

Received: 05 December 2016 Accepted: 09 May 2017 Published: 29 May 2017

Citation:

Cao L, Xia B, Maysam O, Li J, Xie H and Birbaumer N (2017) A

Synchronous Motor Imagery Based Neural Physiological Paradigm for Brain Computer Interface Speller.

Front. Hum. Neurosci. 11:274. doi: 10.3389/fnhum.2017.00274
Brain Computer Interface (BCl) speller is a typical $\mathrm{BCl}$-based application to help paralyzed patients express their thoughts. This paper proposed a novel motor imagery based $\mathrm{BCl}$ speller with Oct-o-spell paradigm for word input. Furthermore, an intelligent input method was used for improving the performance of the $\mathrm{BCl}$ speller. For the English word spelling experiment, we compared synchronous control with previous asynchronous control under the same experimental condition. There were no significant differences between these two control methods in the classification accuracy, information transmission rate (ITR) or letters per minute (LPM). And the accuracy rates of over $70 \%$ validated the feasibility for these two control strategies. It was indicated that Ml-based synchronous control protocol was feasible for $\mathrm{BCl}$ speller. And the efficiency of the predictive text entry (PTE) mode was superior to that of the Non-PTE mode.

Keywords: brain computer interface (BCl), motor imagery (MI), Oct-o-spell paradigm, synchronous control, speller

\section{INTRODUCTION}

Brain-computer interface (BCI) system builds a communication bridge between the brain and the external world by transforming neural signals into control commands without body movement (Birbaumer et al., 1999; Pfurtscheller et al., 2000; Guger et al., 2003; Blankertz et al., 2004; Birbaumer, 2006; Wolpaw, 2014). For severely disabled patients, BCI can help them to manipulate external devices for communication (Sellers and Donchin, 2006; Nijboer et al., 2008; Mainsah et al., 2015).

Electroencephalogram (EEG) is commonly used for BCI. This technology is developed based on neurophysiological patterns such as event related potentials (ERPs) (Blankertz et al., 2011; Zhang et al., 2012; Jin et al., 2014; Yeom et al., 2014), slow cortical potentials (Mensh et al., 2004; Kübler and Birbaumer, 2008),event-related desynchronization/synchronization (Pfurtscheller and Neuper, 2006; Pfurtscheller et al., 2010; Lisi et al., 2014; Sandhya et al., 2014) and steady-state evoked potentials (SSVEPs) (Müller-Putz and Pfurtscheller, 2008; Wang et al., 2008; Allison et al., 2010). BCI speller is one of the common BCI-based applications that paralyzed patients use to express their thoughts (Allison and Pineda, 2003; Krusienski et al., 2008). In 1988, Farwell et al. proposed the P300-based BCI speller (Farwell and Donchin, 1988). P300 signal is detected using Oddball paradigm (Polich and Kok, 1995). The classical interface was composed of a $6 \times 6$ letter matrix including 26 characters, 9 numbers, and a SPACE symbol. Users can spell characters using P300-based target selection. In recent years, P300 spellers have been studied for improving signal processing (Kindermans et al., 2012; Throckmorton et al., 2013; Mainsah et al., 2014a; Speier et al., 2014, 2015) 
and paradigm designing (Combaz et al., 2012; Jin et al., 2012; Mainsah et al., 2014b; Shih et al., 2014; Li et al., 2015). Letter matrices were used to display optional targets, and different flash patterns were developed for improving P300-based classification accuracy and speed.

Cecotti proposed a SSVEP-based speller with five stimulus buttons including character groups (Cecotti, 2010). To spell a letter, user should focus on the corresponding stimuli. Volosyak et al. developed a SSVEP speller controlled using a virtual keyboard (Volosyak et al., 2011). Five commands were used to move the cursor and select the character in a virtual keyboard. Speed of the SSVEP-based speller is reduced due to the limitation of control commands. To solve this problem, 30 flicking stimulus with different frequencies were designated to the speller (Hwang et al., 2012). The average speed was 9.39 letter per minute (LPM), and the average rate of data transfer was 40.72 bits per minute. One disadvantage for these spellers is visual fatigue caused by prolonged gazing.

The Berlin BCI research group has proposed a novel speller paradigm called "Hex-o-Spell," with which subjects spell a character using two-step commands in a hexagon interface (Blankertz et al., 2006). For most subjects, the speed of their performance in this two-class MI-based BCI speller was between 4.6 and 7.6 LPM. However, the performances of existing

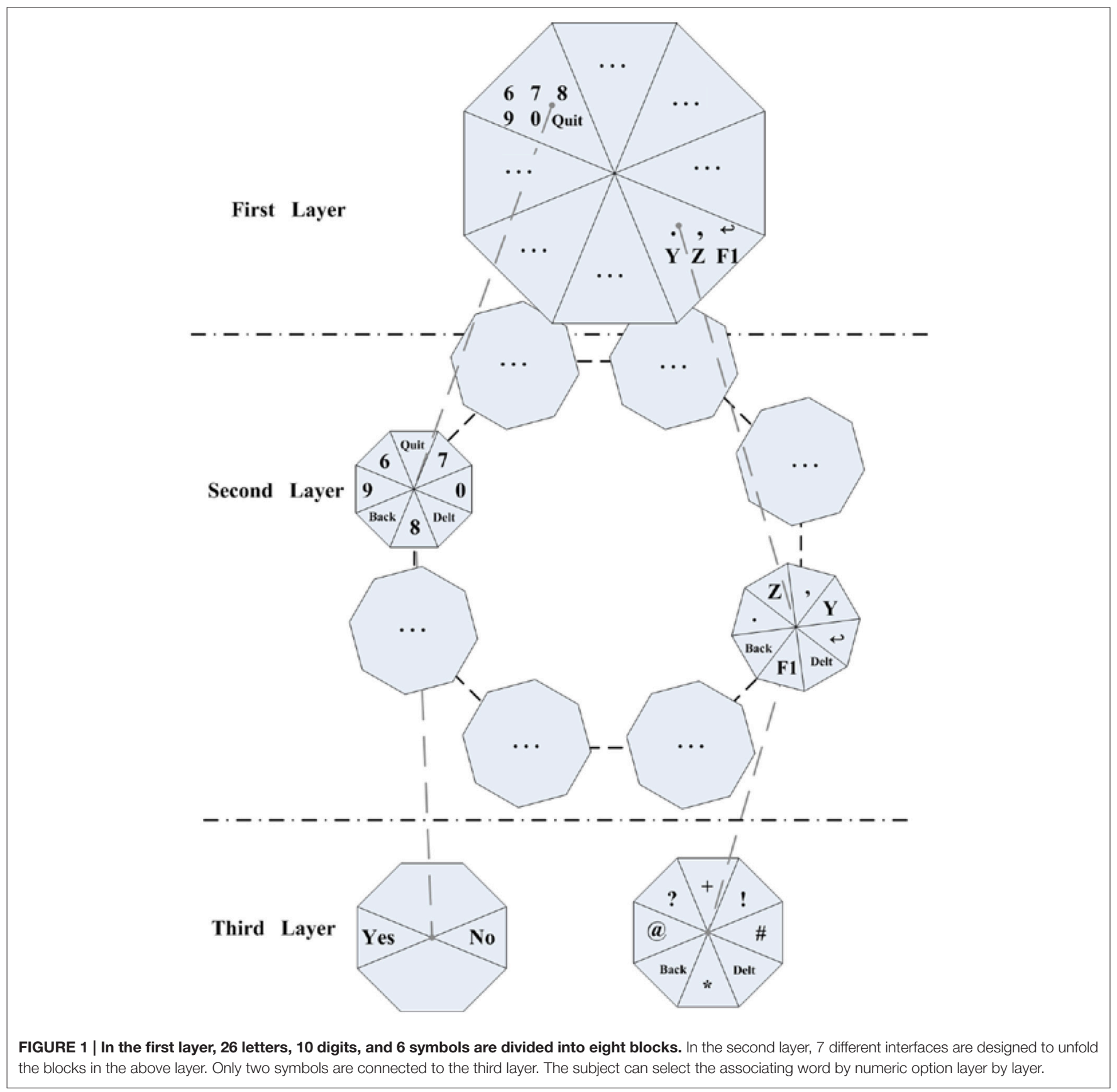




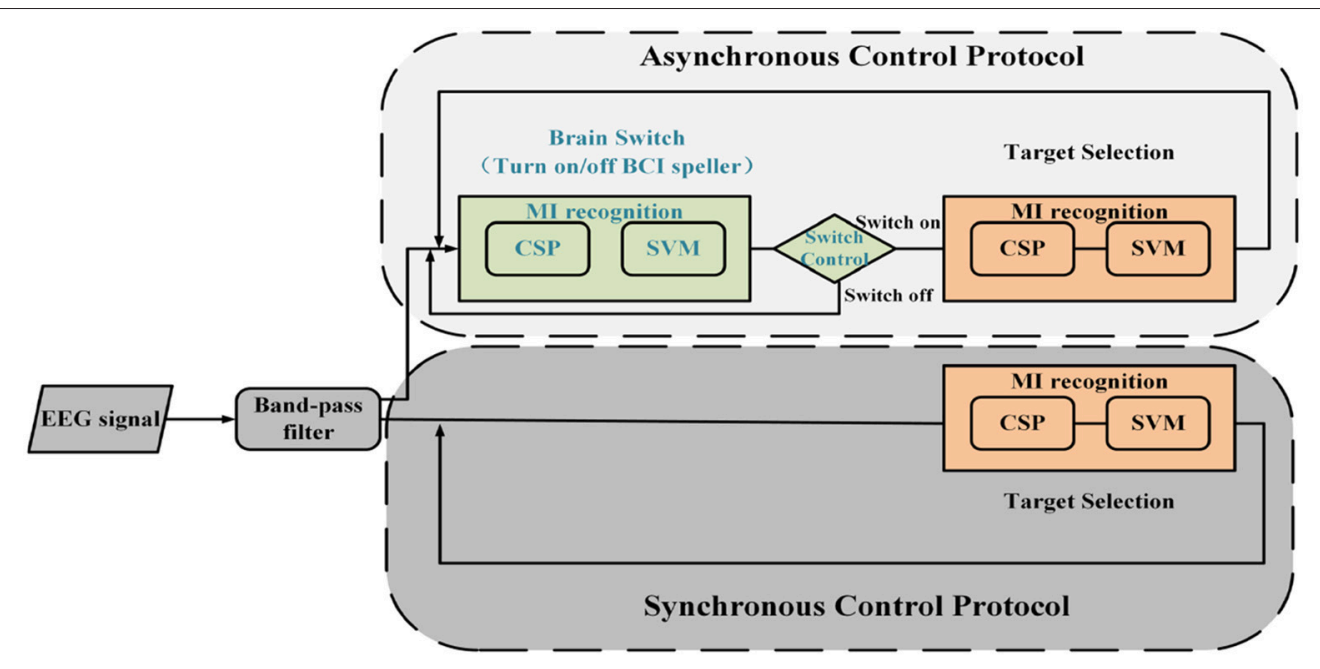

FIGURE 2 | Framework for asynchronous and synchronous control protocols. First, EEG signals are band pass filtered at 5-30 Hz for removing background noise. Then, common spatial pattern (CSP) and support vector machine (SVM) are combined to discriminate three-class (left hand, right hand, foot) MI patterns. For asynchronous control protocol, a brain switch is used for turn on/off this $\mathrm{BCl}$ speller by MI tasks.

MI-based spellers are not comparable with visual stimuli-based speller (Blankertz et al., 2006; D'albis et al., 2012). Recently, the efficiency of MI-based BCI speller has been greatly improved by the Oct-o-spell paradigm in our previous work (Chen et al., 2013). We developed a 2-D cursor control strategy using the modified Hex-o-Spell paradigm and an asynchronous control switch was used for starting the system.

In this paper, synchronous protocol is presented for BCI speller. Furthermore, an intelligent input method is used for improving the performance of the BCI speller. The online experiment is conducted involving three subjects. The comparable result is showed to validate the feasibility of this BCI speller.

\section{METHODS}

\subsection{Systematical Structure}

\subsubsection{Oct-o-Spell Paradigm}

In this paper, a three-layer interface of Oct-o-Spell paradigm is proposed for character input (Figure 1). In the first layer, 26 letters, 10 digits and 6 symbols ("Quit," "=," “ $\leftarrow$," “.," "F1," "Chn/Fn") are divided into eight blocks. In the second layer, 8 different interfaces are designed to unfold the blocks in the first layer. Only two symbols are connected to the third layer. One is "F1," including 6 punctuation: “*," “@," “?," “+," “!,” and“\#.” The other one is 'Quit', which is used for quitting the system. After the user inputs several characters, associative words will be listed by numeric labels. The user can select it by entering the corresponding number. Then, he/she doesn't need to type all letters for saving considerable time. The function is adopted for predictive text entry (PTE) to improve the efficiency of character input. The monitor resolution is $1,440^{*} 838$ pixels and the ratio of the size of cursor target circle and the screen is 0.0003:0. 0065:1.

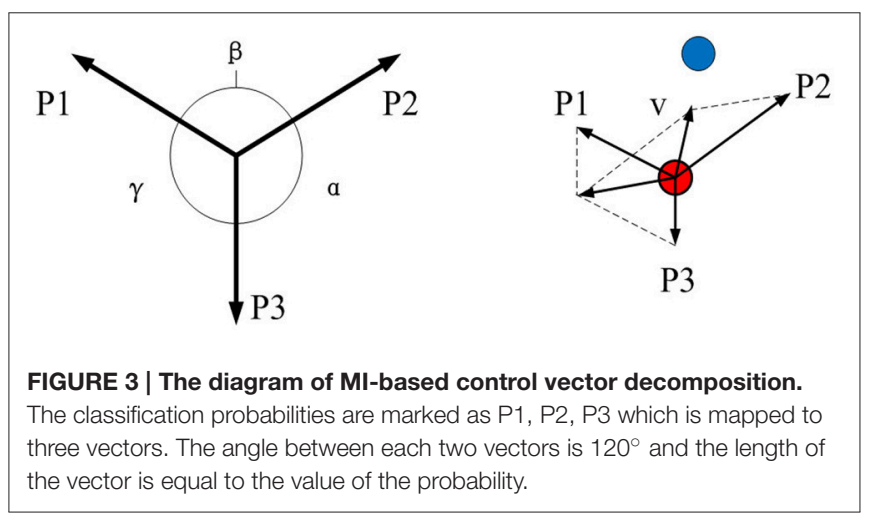

\subsubsection{Motor Imagery-Based Synchronous Control Protocol}

In the previous study (Chen et al., 2013), we presented a motor imagery-based BCI for asynchronous 2D cursor control. Compared with this method, synchronous control remove the brain-actuated switch to improve the efficiency. These two control protocols are illustrated in Figure 2. First, EEG signals are band pass filtered at $5-30 \mathrm{~Hz}$ for removing background noise. Then, common spatial pattern (CSP) and support vector machine (SVM) are combined to discriminate three-class (left hand, right hand, foot) MI patterns. The CSP method is useful for discriminating two populations of EEG. Based on the dataset, a transformation matrix is calculated for a spatial filter. The first and last rows of the CSP transformation matrix could maximize the difference of two groups of data. In our algorithm, the first and last two rows are selected to construct the transformation matrix for further data analysis. On the other hand, the probabilities from SVM classifier are three decimals ranged from 0 to 1 and sum of them equals to 1 . The feature vectors are fed into the SVM classifier, provided by LIBSVM 


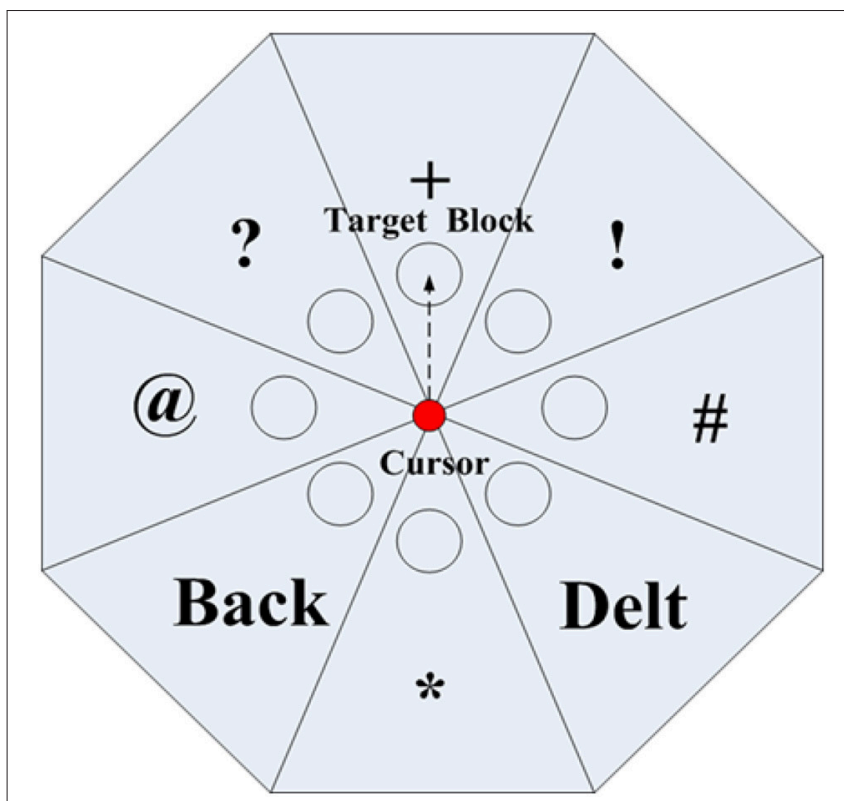

FIGURE 4 | The user performs MI tasks to drive the cursor to the circle of the target block. The corresponding character will be selected for output.

toolbox (Chang and Lin, 2011), to predict the class label and probability estimate for multi-class task. The radial basis function (RBF) is selected as the kernel function and a five-fold crossvalidation in training data is used for choosing suitable SVM parameters. As shown in Figure 3, they are marked as P1, P2 and P3 which are mapped to three vectors. The angle between each two vectors is $120^{\circ}$ and the length of the vector is equal to the value of the probability. To hit the target located between two vectors, the user performs two corresponding motor imagery tasks simultaneously to generate a resultant vector, by which the cursor is moved directly to the target.

On the other hand, the moving distance is computed by the classification result. We define the moving step as L, and the horizontal and vertical moving distances, $d_{x}$ and $d_{y}$, are calculated as follows:

$$
\begin{array}{r}
d_{x}=(p 2-p 1) \times \cos 30^{\circ} \times L \\
d_{y}=\left[(p 1+p 2) \times \sin 30^{\circ}-p 3\right] \times L
\end{array}
$$

where the negative value means that the cursor moves left and vice visa.

To spell character or exit this system, the subject needs to move the cursor to the target circle of the block in each layer. At the first layer, the user can move cursor to reach the target circle of the characters block (containing the target character). Then the interface will extend to the second layer according to the previous selection. Then, the target character can be targeted in the second layer and the speller will output this letter. Only "Quit" or "F1" is chosen in this layer, the interface would extend to the next layer. And the target character can be typed in the third layer or quit the system. To hit a target block (Figure 4), the user should perform MI tasks to drive the cursor to the circle of the target block. If a
TABLE 1 | The Chinese words and their translations used for spelling

\begin{tabular}{|c|c|c|c|c|}
\hline How are you & Thirst & $\begin{array}{l}\text { Do you have a } \\
\text { meal }\end{array}$ & Good morning & Sorry \\
\hline 你好吗 & 渴了 & 吃了吗 & 早上好 & 对不起 \\
\hline $\begin{array}{l}\text { Good night } \\
\text { 晚上好 }\end{array}$ & $\begin{array}{l}\text { Never mind } \\
\text { 没关系 }\end{array}$ & $\begin{array}{l}\text { Thank you } \\
\text { 谢谢 }\end{array}$ & $\begin{array}{l}\text { Remember } \\
\text { 记住了 }\end{array}$ & $\begin{array}{l}\text { Cannot hear } \\
\text { 听不见 }\end{array}$ \\
\hline
\end{tabular}
experiment.

TABLE 2 | The spelling results between Non-PTE mode and PTE mode for all 3 subjects.

\begin{tabular}{lccccccr}
\hline Mode & \multicolumn{3}{c}{ Non-PTE } & & \multicolumn{3}{c}{ PTE } \\
\cline { 2 - 5 } \cline { 7 - 8 } Indicator & ACC (\%) & ITR & LPM & & ACC (\%) & ITR & LPM \\
\hline Sub 1 & 100 & 90.38 & 15.05 & & 95.2 & 56.86 & 15.62 \\
Sub 2 & 94.0 & 53.62 & 6.33 & & 95.5 & 59.13 & 6.77 \\
Sub 3 & 99.0 & 63.49 & 7.6 & & 99.2 & 71.19 & 8.07 \\
Mean & 98.3 & 69.16 & 9.66 & & 96.6 & 62.39 & 10.15 \\
\hline
\end{tabular}

wrong block is selected, the "Back" command in the next layer can be selected to return to the previous interface. If a wrong character is chose, the subject can select the "Delt" command to delete it in the second layer. The MI BCI system will produce projection values for cursor moving every $0.5 \mathrm{~s}$. Typically, the subject needs to take about $3 \mathrm{~s}$ (6 trials) to conduct MI task for targeting the circle of a block in one certain layer. And the maximum time consumption will be given in the description of experimental settings.

\subsection{Subjects and Experimental Settings}

Six healthy subjects ( 4 males, 2 females) aged between 19 and 26 , took part in our experiment. All of them were naive in BCI and consent to be involved in the study. Firstly, three-class (left hand imagery, right hand imagery and feet imagery) MI sessions were conducted for training in one day. Each training session included 20 trials for each class and each trial last 2 s. Subjects had a short break between sessions. In the experiment, each subject was seated in front of a notebook computer, with arms on the chair arms and hands relaxed. An arrow pointing three different directions was used to indicate one class MI. Finally, three subjects who had achieved performance rates of over $70 \%$ (criterion level) were selected for subsequent spelling experiment. In the spelling experiment, all previous train data were used for training SVM classifiers. EEG signals were recorded by a 16-channel g.USBamp amplifier, and 13 electrodes were placed according to the international 10-20 system (FC3, FCZ, FC4, C5, C3, C1, CZ, C2, C4, C6, CP3, CPZ, and CP4).The ground and reference electrodes were placed on the $\mathrm{FZ}$ position and the right earlobe. EEG signals were sampled at $256 \mathrm{~Hz}$ and band-pass filtered between 5 and $30 \mathrm{~Hz}$.

Online copy spelling experiment: In this experiment, the subject used the above synchronous method for MI-based BCI task. This BCI system could produce three projection values in corresponding directions every $0.5 \mathrm{~s}$ for cursor moving. The BCI classifier model was trained by previous MI training sessions. 


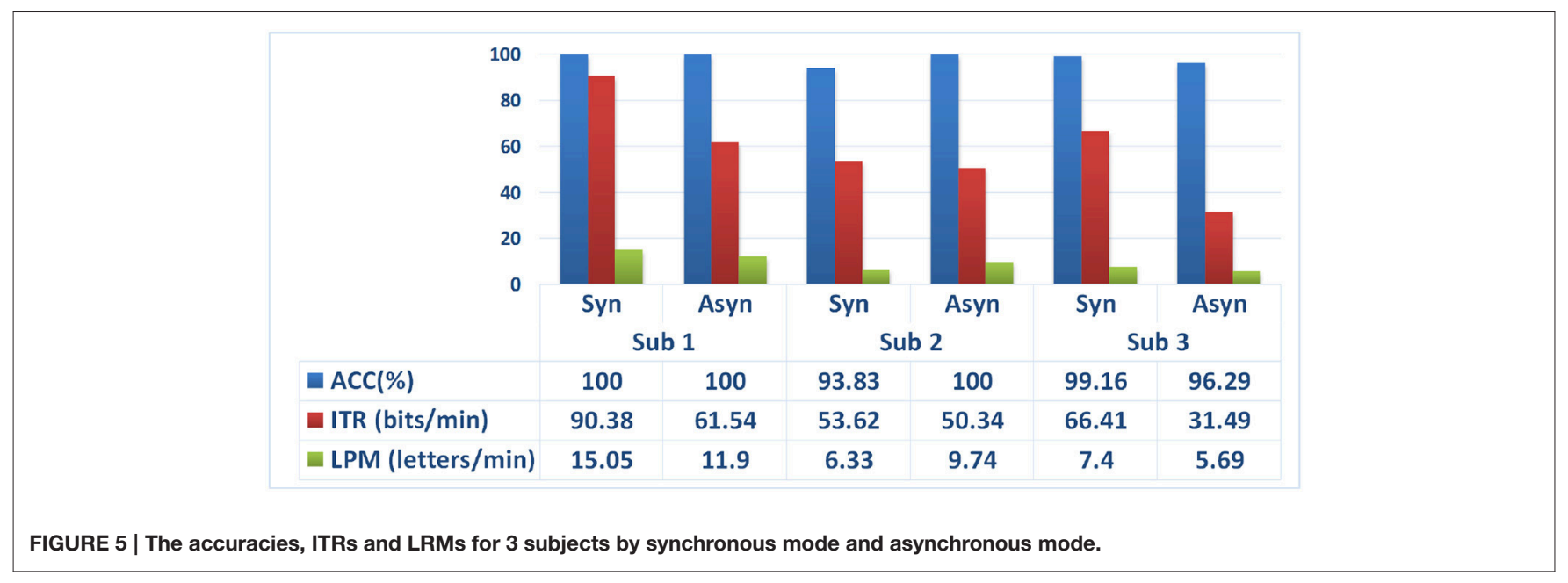

TABLE 3 | The experimental performance of Chinese words spelling for 3 subjects.

\begin{tabular}{lccc}
\hline Indicator & Accuracy (\%) & LPM & ITR \\
\hline Sub 1 & 82.82 & 5.41 & 34.76 \\
Sub 2 & 94.94 & 5.22 & 48.02 \\
Sub 3 & 98.65 & 7.4 & 66.41 \\
Mean & $92.13 \%$ & 6.01 & 49.73 \\
\hline
\end{tabular}

In order to help the subjects with the speller interface, each subject performed 20 free spell familiarize runs in which subject was asked to type characters from A to Z (26 letters) for 2 days. Then the subjects participated in an online copy spelling experiment on the third day. Firstly, 3 subjects spelled 15 English words by PTE and non-PTE modes. The words were selected from reference (Hwang et al., 2012): "WOMEN, DESK, WATER, HAND, MEMORY, ZONE, BABY, FACE, TAXI, JUNE, QUICK, VIDEO, GOLF, HOUR, PENCIL" for synchronous paradigms testing. There was a 3-s interval between trials. If the subject couldn't spell the word correctly in $40 \mathrm{~s}$, this trial would be ended and another trial started. Wrongly typed letters had to be corrected. Furthermore, all subjects performed Chinese character input by PTE mode. Ten common words were used for this test (Table 1). And the rule was the same as English character input.

\section{RESULTS}

In our study, online English and Chinese words spelling experiments were respectively conducted to evaluate our synchronous BCI system. Conventionally, some typical indicators were selected to assess the systematical efficiency. (1) Accuracy rate (ACC): the percentage of successful classification result. (2) Information transmission ratio (ITR): the bit rate of information communicated per unit time. (3) Letter per minute (LPM).

Table 2 shows the performance for 3 subjects. The comparison between PTE and non-PTE methods didn't show significant differences.

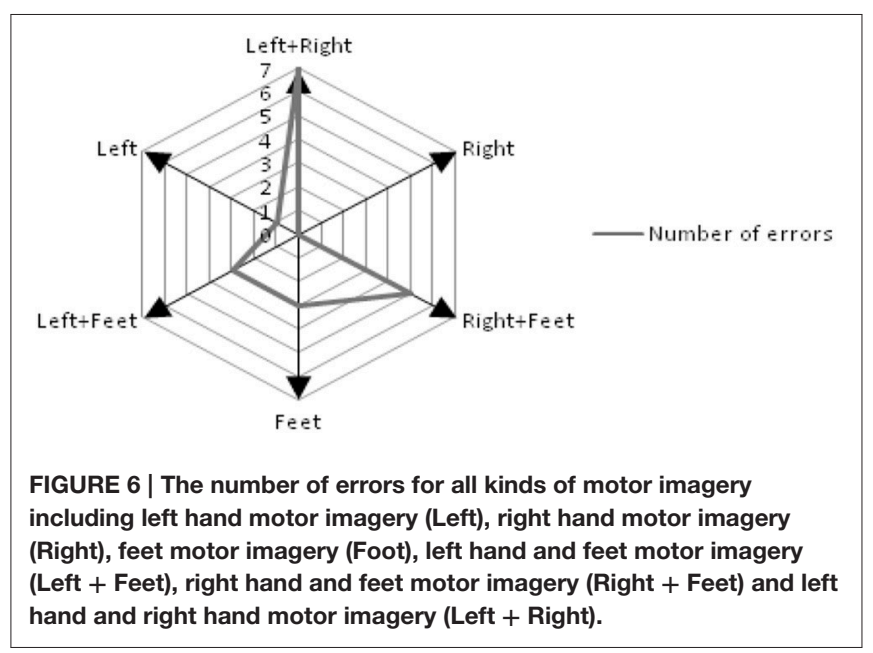

For the English word spelling experiment, we compared synchronous control with previous asynchronous control under the same experimental condition (Figure 5). There were no significant differences between these two control methods in ACC (paired $t$-test: $t=0.4125, p>0.05)$, ITR (paired $t$-test: $t=$ 2.3053, $p>0.05$ ) or LPM (paired $t$-test: $t=0.2428, p>0.05$ ). Even so, the synchronous mode had better performance in ITR and LPM. It was verified that this strategy had higher efficiency in BCI output than that of asynchronous mode.

Chinese spelling tasks were performed by all subjects. The performance is presented in Table 3. All subjects achieved sufficient performance rate of over $70 \%$ (criterion level). It demonstrated that our proposed BCI speller was feasible for both English and Chinese.

\section{DISCUSSION}

For 3 subjects, experimental results showed that motor imagery was feasible for common communication and expression in BCI. All subjects gave positive feedback to the manipulation 
TABLE 4 | The detailed performance of character input for Subject 1.

\begin{tabular}{|c|c|c|c|c|}
\hline \multirow{2}{*}{$\begin{array}{l}\text { Mode } \\
\text { Word }\end{array}$} & \multicolumn{2}{|c|}{ Non-PTE } & \multicolumn{2}{|c|}{ PTE } \\
\hline & \multicolumn{4}{|c|}{ Performance of Subject 1} \\
\hline women & women & women & $\leftarrow$ wo4 & $\leftarrow$ wo4 \\
\hline desk & desk & desk & des4 & des4 \\
\hline water & water & water & wa2 & $r \leftarrow w a 2$ \\
\hline hand & hand & hand & ha5 $\leftarrow \leftarrow \leftarrow$ ha 6 & ha(back) 6 \\
\hline memory & memory & memory & me2 & me2 \\
\hline zone & zone & zone & z3 & z3 \\
\hline baby & baby & baby & ba2 & ba2 \\
\hline face & face & face & $\mathrm{fa} 4$ & $\mathrm{fa} 4$ \\
\hline taxi & taxi & taxi & $\mathrm{r} \leftarrow \mathrm{h} \leftarrow \operatorname{tax} 3$ & taxi1 \\
\hline june & june & june & ju4 & j(back)9 \\
\hline quick & quick & quick & q3 & q3 \\
\hline video & video & video & v1 & $\mathrm{v} 1$ \\
\hline golf & golf & golf & 97 & 97 \\
\hline hour & hour & hour & hou3 & hou3 \\
\hline pencil & pencil & pencil & pen6 & pen6 \\
\hline Mean accuracy & $100 \%$ & $100 \%$ & $93.64 \%$ & $96.72 \%$ \\
\hline LPM(letters/min) & 14.53 & 15.56 & 15.43 & 15.79 \\
\hline ITR(bits/min) & 87.39 & 93.36 & 55.55 & 58.17 \\
\hline $\begin{array}{l}\text { Average number of } \\
\text { spelling letters per word }\end{array}$ & 4.53 & 4.53 & 3.73 & 3.13 \\
\hline
\end{tabular}

' $\leftarrow$ ' means $\cdot$ Delt'.

of the speller after experiments. No pronounced difference was observed between our MI-based speller and other BCI spellers (e.g., SSVEP, P300) (Cecotti, 2010; Jin et al., 2011a,b; Xu et al., 2014). Moreover, the accuracy and ITR of our MI-based speller are approximately equal to those of hybrid BCIs (Xu et al., 2013; Yin et al., 2013). This MI-based control strategy is useful for Oct$\mathrm{o}$-Spell paradigm. The result of LPM implied that our MI-control strategy had better output performance than those of previous BCI spellers (Blankertz et al., 2006; D'albis et al., 2012). Moreover, the number of candidate characters was 36 for our BCI system. And previous MI-based BCI spellers had few target characters for user selection. It indicated that our $\mathrm{BCI}$ speller was more practical than other MI-based BCI spellers.

In the experiment, subjects performed two motor imagery tasks simultaneously. Compared with traditional single modalitybased MI control task, it was more difficult to obtain accurate results in this parallel control system because of simultaneous multi modality -based MIs. We reported the number of errors for all motor imagery tasks (Figure 6). The error was defined as the incorrect block selection resulted from cursor moving, which indicated by the use of "Back" and "Delt." It was speculated that, before these two characters were used for correcting a mistake, the user didn't move the cursor along with the target direction, caused by poor performance in MI tasks. It indicated that synchronous motor imagery tasks (i.e., left hand and feet motor imagery, right hand and feet motor imagery, and left hand and right hand motor imagery) were harder to be mentally controlled. Hand-based motor imagery with two hands was the
TABLE 5 | The detailed performance of character input for Subject 2.

\begin{tabular}{|c|c|c|c|c|}
\hline \multirow{2}{*}{$\begin{array}{l}\text { Mode } \\
\text { Word }\end{array}$} & \multicolumn{2}{|c|}{ Non-PTE } & \multicolumn{2}{|c|}{ PTE } \\
\hline & & Performan & e of Subject 2 & \\
\hline women & women & women & w(back)o4 & $3 \leftarrow w 04$ \\
\hline desk & des(back)k & desk & (back)des4 & $\begin{array}{l}\operatorname{desk}(\text { back) } \\
\text { (back) } 4\end{array}$ \\
\hline water & water & water & wa2 & wa2 \\
\hline hand & (back)hand & hand & ha6 & $\begin{array}{l}\text { ha } \leftarrow \text { (back) } \\
\text { and1 }\end{array}$ \\
\hline memory & $\mathrm{md} \leftarrow$ emory & memory & me2 & me2 \\
\hline zone & $\leftarrow$ zone & zone & zo2 & z3 \\
\hline baby & baby & baby & ba2 & ba2 \\
\hline face & face & face & fa4 & fac2 \\
\hline taxi & $\operatorname{taxj} \leftarrow i$ & taxi & $\operatorname{tax} 3$ & $\operatorname{tax} 3$ \\
\hline june & (back)june & june & ju4 & (back)ju4 \\
\hline quick & quick & quicl $\leftarrow k$ & q3 & q3 \\
\hline video & $\mathrm{s} \leftarrow$ video & video & $\mathrm{vbn} \leftarrow \leftarrow$ (back) 1 & v1 \\
\hline golf & $\mathrm{gol} \leftarrow \leftarrow \mathrm{j} \leftarrow$ olf & golf & go3 & go3 \\
\hline hour & hour & (back)hour & hou3 & (back)hou3 \\
\hline pencil & pencif $\leftarrow 1$ & pencil & pen6 & pen6 \\
\hline Mean accuracy & $89.94 \%$ & $97.71 \%$ & $96.12 \%$ & $94.96 \%$ \\
\hline LPM(letters/min) & 5.14 & 7.52 & 6.35 & 7.18 \\
\hline ITR(bits/min) & 50.46 & 56.77 & 69.87 & 48.37 \\
\hline $\begin{array}{l}\text { Average number } \\
\text { of spelling letters } \\
\text { per word }\end{array}$ & 5.73 & 4.67 & 3.4 & 3.6 \\
\hline
\end{tabular}

' $\leftarrow$ ' means . Delt'.

most difficult task for moving the cursor to the target point. Therefore, characters used frequently must be located in the position where the subject is able to move the cursor by single modality-based MI.

Although the precision and ITR of Non-PTE mode was slightly higher than PTE mode, no significant differences were observed in terms of performance between the PTE and the Non-PTE mode. The detailed process of character input for all subjects was reported at Tables 4-6. Although there were more spelling errors in the PTE mode, which was probably due to distraction caused by word selection, the number of required characters by the PTE mode was less than that for the NonPTE mode. Nevertheless, this disadvantage was compensated by the reduction of required spelling letters. It was reflected in the average number of spelling letters per word. That was the reason why the LPM of the PTE mode was higher than that for the Non-PTE mode. Moreover, the efficiency of PTE mode could be improved by raising the precision of character selection.

The ITRs for the synchronous mode were higher than those for the asynchronous mode for all the subjects. The asynchronous BCI took some time to start the speller, which reduced the transmission efficiency in a trial. Therefore, the number of switch control was decreased for improving the efficiency of the BCI speller. Therefore, subjects needed to reduce the use of switch control for improving the efficiency of the BCI speller. For instance, when the user activated the switch consciously in the 
TABLE 6 | The detailed performance of character input for Subject 3.

\begin{tabular}{|c|c|c|c|c|}
\hline \multirow{2}{*}{$\begin{array}{l}\text { Mode } \\
\text { Word }\end{array}$} & \multicolumn{2}{|c|}{ Non-PTE } & \multicolumn{2}{|c|}{ PTE } \\
\hline & \multicolumn{4}{|c|}{ Performance of Subject 3} \\
\hline women & women & women & wo4 & wo4 \\
\hline desk & desk & desk & des4 & des4 \\
\hline water & water & water & wa2 & wa2 \\
\hline hand & han(back)d & hand & ha6 & h(back)a6 \\
\hline memory & memory & memor $\leftarrow r y$ & m(back)e2 & me2 \\
\hline zone & zone & zone & z3 & z3 \\
\hline baby & baby & baby & ba2 & ba2 \\
\hline face & face & face & fa4 & fa4 \\
\hline $\operatorname{taxi}$ & taxi & taxi & $\mathrm{r} \leftarrow \mathrm{h} \leftarrow \operatorname{tax} 3$ & $\operatorname{taxi1}$ \\
\hline june & june & june & ju4 & jun2 \\
\hline quick & quick & quick & q3 & qu3 \\
\hline video & video & video & $\mathrm{v} 1$ & $\mathrm{v} 1$ \\
\hline golf & golf & golf & $\mathrm{g}($ back $) 7$ & g(back)o3 \\
\hline hour & hour & hour & hou3 & hou3 \\
\hline pencil & pencil & pencil & pen6 & pen6 \\
\hline Mean Accuracy & $99.33 \%$ & $99.05 \%$ & $96.12 \%$ & $98.33 \%$ \\
\hline LPM(letters/min) & 6.01 & 9.19 & 6.55 & 8.58 \\
\hline ITR(bits/min) & 62.39 & 64.57 & 72.41 & 69.96 \\
\hline $\begin{array}{l}\text { Average number of } \\
\text { spelling letters per word }\end{array}$ & 4.53 & 4.67 & 3.13 & 3.2 \\
\hline
\end{tabular}

' $\leftarrow$ ' means · Delt'.

first layer, the switch would be active automatically for target selection in the second and third layer. Because the user intended to spell something after activating the speller in the first layer. The switch was redundant to detect mental state when the user was conscious to manipulating. Thus, the time consumption would be reduced for better ITR and LPM. Meanwhile, we could add a selective function for switching off the system in the second and third layers. It was used for correcting the false positive selection in the first layer. This control protocol that possess the superiority of both patterns, would be developed in further work.

The number of electrodes of our BCI speller was higher than SSVEP-based BCI speller (Xu et al., 2014). As mentioned,

\section{REFERENCES}

Allison, B., Lüth, T., Valbuena, D., Teymourian, A., Volosyak, I., and Gräser, A. (2010). BCI demographics: how many (and what kinds of) people can use an ssvep BCI? Neural Syst. Rehabil. Eng. IEEE Trans. 18, 107-116. doi: 10.1109/TNSRE.2009.2039495

Allison, B. Z., and Pineda, J. (2003). Erps evoked by different matrix sizes: implications for a brain computer interface (BCI) system. Neural Syst. Rehabil. Eng. IEEE Trans. 11, 110-113. doi: 10.1109/TNSRE.2003.814448

Birbaumer, N. (2006). Breaking the silence: brain-computer interfaces (BCI) for communication and motor control. Psychophysiology 43, 517-532. doi: $10.1111 / j .1469-8986.2006 .00456 . x$

Birbaumer, N., Ghanayim, N., Hinterberger, T., Iversen, I., Kotchoubey, B., Kübler, A., et al. (1999). A spelling device for the paralysed. Nature 398, 297-298. doi: $10.1038 / 18581$ fewer channels were beneficial for wearable portable BCI devices. And the selection of electrodes would be considered for real conditions and user experience in future. Furthermore, optional mode-based BCI speller should be developed to account for individual differences.

\section{CONCLUSIONS}

This paper proposed a novel MI-based BCI speller with Octo-spell paradigm for word input. The 3D motor imagery was used for synchronous control. The detailed analysis was given in the discussion. Our results showed that MI-based synchronous protocol was feasible for BCI speller. And the efficiency of the PTE mode was superior to that of the non-PTE mode.

\section{ETHICS STATEMENT}

This study was carried out in accordance with the recommendations of "name of guidelines, name of committee" with written informed consent from all subjects. All subjects gave written informed consent in accordance with the Declaration of Helsinki. The protocol was approved by the "name of committee."

\section{AUTHOR CONTRIBUTIONS}

LC and BX contributed to the conception of the study, as well as performed the data analyses and wrote the manuscript. OM, $\mathrm{JL}$, and HX contributed significantly to analysis and manuscript preparation. And NB helped revising the manuscript critically for important intellectual content.

\section{ACKNOWLEDGMENTS}

The work was supported by the Fundamental Research Funds for International Young Scientists Title of the awarded research project (Grant No. 61550110252) and Shanghai NSF research project (Grant No. 16JC1401300).

Blankertz, B., Dornhege, G., Krauledat, M., Schröder, M., Williamson, J., MurraySmith, R., et al. (2006). "The berlin brain-computer interface presents the novel mental typewriter hex-o-spell," in Proceedings of the 3rd International Brain Computer Interface Workshop and Training Course (Graz).

Blankertz, B., Lemm, S., Treder, M., Haufe, S., and Müller, K.-R. (2011). Single-trial analysis and classification of erp componentsa tutorial. Neuroimage 56, 814-825. doi: 10.1016/j.neuroimage.2010. 06.048

Blankertz, B., Müller, K.-R., Curio, G., Vaughan, T. M., Schalk, G., Wolpaw, J. R., et al. (2004). The BCI competition 2003: progress and perspectives in detection and discrimination of eeg single trials. Biomed. Eng. IEEE Trans. 51, 1044-1051. doi: 10.1109/TBME.2004.826692

Cecotti, H. (2010). A self-paced and calibration-less ssvep-based brain-computer interface speller. Neural Syst. Rehabil. Eng. IEEE Trans. 18, 127-133. doi: 10.1109/TNSRE.2009.2039594 
Chang, C.-C., and Lin, C.-J. (2011). Libsvm: a library for support vector machines. ACM Trans. Intell. Syst. Technol. (TIST) 2:27. doi: 10.1145/1961189.1961199

Chen, C., Yang, J., Huang, Y., Li, J., and Xia, B. (2013). "A cursor control based chinese-english BCI speller," in Neural Information Processing, eds M. Lee, A. Hirose, Z.-G. Hou, and R. M. Kil (Dageu: Springer), 403-410.

Combaz, A., Chumerin, N., Manyakov, N. V., Robben, A., Suykens, J. A., and Van Hulle, M. M. (2012). Towards the detection of error-related potentials and its integration in the context of a p300 speller braincomputer interface. Neurocomputing 80, 73-82. doi: 10.1016/j.neucom.2011. 09.013

D’albis, T., Blatt, R., Tedesco, R., Sbattella, L., and Matteucci, M. (2012). A predictive speller controlled by a brain-computer interface based on motor imagery. ACM Trans. Comput. Hum. Inter. (TOCHI) 19:20. doi: $10.1145 / 2362364.2362368$

Farwell, L. A., and Donchin, E. (1988). Talking off the top of your head: toward a mental prosthesis utilizing event-related brain potentials. Electroencephal. Clin. Neurophysiol. 70, 510-523.

Guger, C., Edlinger, G., Harkam, W., Niedermayer, I., and Pfurtscheller, G. (2003). How many people are able to operate an eeg-based braincomputer interface (BCI)? Neural Syst. Rehabil. Eng. IEEE Trans. 11, 145-147. doi: 10.1109/TNSRE.2003.814481

Hwang, H.-J., Lim, J.-H., Jung, Y.-J., Choi, H., Lee, S. W., and Im, C.-H. (2012). Development of an ssvep-based BCI spelling system adopting a qwerty-style led keyboard. J. Neurosci. Methods 208, 59-65. doi: 10.1016/j.jneumeth.2012.04.011

Jin, J., Allison, B. Z., Sellers, E. W., Brunner, C., Horki, P., Wang, X., et al. (2011a). An adaptive p300-based control system. J. Neural Eng. 8:036006. doi: $10.1088 / 1741-2560 / 8 / 3 / 036006$

Jin, J., Allison, B. Z., Sellers, E. W., Brunner, C., Horki, P., Wang, X., et al. (2011b). Optimized stimulus presentation patterns for an event-related potential eeg-based brain-computer interface. Med. Biol. Eng. Comput. 49, 181-191. doi: 10.1007/s11517-010-0689-8

Jin, J., Allison, B. Z., Zhang, Y., Wang, X., and Cichocki, A. (2014). An erp-based BCI using an oddball paradigm with different faces and reduced errors in critical functions. Int. J. Neural Syst. 24:1450027. doi: $10.1142 / S 0129065714500270$

Jin, J., Sellers, E. W., and Wang, X. (2012). Targeting an efficient target-to-target interval for p300 speller brain-computer interfaces. Med. Biol. Eng. Comput. 50, 289-296. doi: 10.1007/s11517-012-0868-x

Kindermans, P.-J., Verstraeten, D., and Schrauwen, B. (2012). A bayesian model for exploiting application constraints to enable unsupervised training of a p300-based BCI. PLoS ONE 7:e33758. doi: 10.1371/journal.pone.00 33758

Krusienski, D. J., Sellers, E. W., McFarland, D. J., Vaughan, T. M., and Wolpaw, J. R. (2008). Toward enhanced p300 speller performance. J. Neurosci. Methods 167, 15-21. doi: 10.1016/j.jneumeth.2007.07.017

Kübler, A., and Birbaumer, N. (2008). Brain-computer interfaces and communication in paralysis: Extinction of goal directed thinking in completely paralysed patients? Clin. Neurophysiol. 119, 2658-2666. doi: 10.1016/j.clinph.2008.06.019

Li, Q., Liu, S., Cao, K., Li, J., Shi, K. Y., and Wu, L. F. (2015). A comparative study on single display and region-based p300 speller paradigms for brain-computer interface. Adv. Mat. Res. 1153-1157.

Lisi, G., Noda, T., and Morimoto, J. (2014). Decoding the erd/ers: influence of afferent input induced by a leg assistive robot. Front. Syst. Neurosci. 8:85. doi: 10.3389/fnsys.2014.00085

Mainsah, B., Collins, L., Colwell, K., Sellers, E., Ryan, D., Caves, K., et al. (2015). Increasing BCI communication rates with dynamic stopping towards more practical use: an als study. J. Neural Eng. 12:016013. doi: 10.1088/1741-2560/12/1/016013

Mainsah, B. O., Morton, K., Collins, L., Sellers, E., and Throckmorton, C. (2014a). Moving away from error-related potentials to achieve spelling correction in p300 spellers. IIEEE Trans. Neural. Syst. Rehabil. Eng. 23, 737-743. doi: 10.1109/TNSRE.2014.2374471

Mainsah, B. O., Colwell, K. A., Collins, L. M., and Throckmorton, C. S. (2014b). Utilizing a language model to improve online dynamic data collection in p300 spellers. Neural Syst. Rehabil. Eng. IEEE Trans. 22, 837-846. doi: 10.1109/TNSRE.2014.2321290
Mensh, B. D., Werfel, J., and Seung, H. S. (2004). BCI competition 2003 data set ia: combining gamma-band power with slow cortical potentials to improve single-trial classification of electroencephalographic signals. Biomed. Eng. IEEE Trans. 51, 1052-1056. doi: 10.1109/TBME.2004. 827081

Müller-Putz, G. R., and Pfurtscheller, G. (2008). Control of an electrical prosthesis with an ssvep-based BCI. Biomed. Eng. IEEE Trans. 55, 361-364. doi: 10.1109/TBME.2007.897815

Nijboer, F., Furdea, A., Gunst, I., Mellinger, J., McFarland, D. J., Birbaumer, N., et al. (2008). An auditory brain-computer interface (BCI). J. Neurosci. Methods 167, 43-50. doi: 10.1016/j.jneumeth.2007. 02.009

Pfurtscheller, G., and Neuper, C. (2006). Future prospects of erd/ers in the context of brain-computer interface (BCI) developments. Progr. Brain Res. 159, 433437. doi: 10.1016/S0079-6123(06)59028-4

Pfurtscheller, G., Neuper, C., Guger, C., Harkam, W., Ramoser, H., Schlogl, A., et al. (2000). Current trends in graz brain-computer interface (BCI) research. IEEE Trans. Rehabil. Eng. 8, 216-219. doi: 10.1109/86. 847821

Pfurtscheller, G., Solis-Escalante, T., Ortner, R., Linortner, P., and Müller-Putz, G. R. (2010). Self-paced operation of an ssvepbased orthosis with and without an imagery-based "brain switch:" a feasibility study towards a hybrid BCI. Neural Syst. Rehabil. Eng. IEEE Trans. 18, 409-414. doi: 10.1109/TNSRE.2010.20 40837

Polich, J., and Kok, A. (1995). Cognitive and biological determinants of p300: an integrative review. Biol. Psychol. 41, 103-146.

Sandhya, B., Shendkar, C., and Mahadevappa, M. (2014). "Single channel event related (de) synchronization (ERD/ERS) analysis of motor execution in stroke affected foot drop subjects," in Medical Imaging, $m$-Health and Emerging Communication Systems (MedCom), 2014 International Conference on (Greater Noida: IEEE), 325-328.

Sellers, E. W., and Donchin, E. (2006). A p300-based brain-computer interface: initial tests by als patients. Clin. Neurophysiol. 117, 538-548. doi: 10.1016/j.clinph.2005.06.027

Shih, J., Townsend, G., Krusienski, D., Shih, K., Shih, R., Heggeli, K., et al. (2014). Comparison of the checkerboard p300 speller vs. the row-column speller in normal elderly and an aphasic stroke population (s21. 006). Neurology 82(10 Suppl.).

Speier, W., Arnold, C., Lu, J., Deshpande, A., and Pouratian, N. (2014). Integrating language information with a hidden markov model to improve communication rate in the p300 speller. Neural Syst. Rehabil. Eng. IEEE Trans. 22, 678-684. doi: 10.1109/TNSRE.2014.2300091

Speier, W., Deshpande, A., and Pouratian, N. (2015). A method for optimizing eeg electrode number and configuration for signal acquisition in p300 speller systems. Clin. Neurophysiol. 126, 1171-1177. doi: 10.1016/j.clinph.2014. 09.021

Throckmorton, C. S., Colwell, K. A., Ryan, D. B., Sellers, E. W., and Collins, L. M. (2013). Bayesian approach to dynamically controlling data collection in p300 spellers. Neural Syst. Rehabil. Eng. IEEE Trans. 21, 508-517. doi: 10.1109/TNSRE.2013.2253125

Volosyak, I., Moor, A., and Gräser, A. (2011). "A dictionary-driven ssvep speller with a modified graphical user interface," in Advances in Computational Intelligence, eds J. Cabestany, I. Rojas, and G. Joya (Malaga: Springer), 353-361.

Wang, Y., Gao, X., Hong, B., Jia, C., and Gao, S. (2008). Brain-computer interfaces based on visual evoked potentials. Eng. Med. Biol. Magazine IEEE 27, 64-71. doi: 10.1109/MEMB.2008.923958

Wolpaw, J. R. (2014). The BCI endeavor and the mission of this new journal. Brain Comp. Inter. 1, 2-4. doi: 10.1080/2326263X.2014.884740

Xu, M., Chen, L., Zhang, L., Qi, H., Ma, L., Tang, J., et al. (2014). A visual parallel-BCI speller based on the time-frequency coding strategy. J. Neural Eng. 11:026014. doi: 10.1088/1741-2560/11/2/026014

Xu, M., Qi, H., Wan, B., Yin, T., Liu, Z., and Ming, D. (2013). A hybrid BCI speller paradigm combining p300 potential and the ssvep blocking feature. J. Neural Eng. 10:026001. doi: 10.1088/1741-2560/10/2/ 026001 
Yeom, S.-K., Fazli, S., Müller, K.-R., and Lee, S.-W. (2014). An efficient erp-based brain-computer interface using random set presentation and face familiarity. PLoS ONE 9:e111157. doi: 10.1371/journal.pone.0111157

Yin, E., Zhou, Z., Jiang, J., Chen, F., Liu, Y., and Hu, D. (2013). A novel hybrid BCI speller based on the incorporation of ssvep into the p300 paradigm. J. Neural Eng. 10:026012. doi: 10.1088/1741-2560/10/2/026012

Zhang, Y., Zhao, Q., Jin, J., Wang, X., and Cichocki, A. (2012). A novel BCI based on erp components sensitive to configural processing of human faces. J. Neural Eng. 9:026018. doi: 10.1088/1741-2560/9/2/ 026018
Conflict of Interest Statement: The authors declare that the research was conducted in the absence of any commercial or financial relationships that could be construed as a potential conflict of interest.

Copyright (C) 2017 Cao, Xia, Maysam, Li, Xie and Birbaumer. This is an open-access article distributed under the terms of the Creative Commons Attribution License (CC $B Y)$. The use, distribution or reproduction in other forums is permitted, provided the original author(s) or licensor are credited and that the original publication in this journal is cited, in accordance with accepted academic practice. No use, distribution or reproduction is permitted which does not comply with these terms. 